\title{
Performance of a data acquisition system for a large germanium detector array
}

\author{
A. Kimura ${ }^{1, a}$, M. Koizumi ${ }^{1}$, Y. Toh ${ }^{1}$, J. Goto $^{2}$, and M. Oshima ${ }^{1}$ \\ 1 Japan Atomic Energy Agency, 2-4 Shirakata-Shirane, Tokai-mura, Ibaraki-ken, Japan \\ 2 Niigata University, 1-757 Asahimachi-dori, Niigata-shi, Niigata-ken, Japan
}

\begin{abstract}
We have evaluated the performance of our new data acquisition system (DAQ), which utilizes digital signal processing techniques and was developed for a $4 \pi$ Ge spectrometer. The total energy resolution of this system was $2.5 \mathrm{keV}$ in FWHM for 1.33-MeV gamma rays and $16.4 \mathrm{keV}$ in FWHM for 10.83-MeV prompt gamma rays. The time resolution of timing signals, which are used for the measurements of coincident gamma rays, was $120 \mathrm{~ns}$ in FWHM. Those values fulfilled our requirements for the DAQ. A method of dead time correction with this system was also developed.
\end{abstract}

\section{Introduction}

The aim of our project is the acquisition of neutron crosssection data for the minor actinides (such as ${ }^{237} \mathrm{~Np},{ }^{241} \mathrm{Am}$ and ${ }^{243} \mathrm{Am}$ ) that are important for the development of innovative nuclear reactor technology. For this purpose we have constructed "the $4 \pi$ Ge spectrometer" which detects cascade gamma rays at the same time emitted in a neutron capture reaction [1]. Such a gamma-gamma or higher-fold gamma coincidence measurement is called as a multiple gamma ray detection method.

In Time Of Flight (TOF) measurements of neutron capture reactions with multiple gamma ray detection method, a DAQ have to handle many signals from $\mathrm{Ge}$ and BGO detectors at high event rate. The DAQ is required to have high time resolution and high speed. At the same time, the DAQ is also required to have reasonable energy resolution at wide range for analyses of discrete gamma rays and to effectively apply energy gates on them. On these requirements, a new data acquisition system with digital signal processing techniques has been developed, and its conceptual design has been presented in the ND2004 conference [2]. In this ND2007, the energy resolution and time resolution is reported.

In order to obtain a neutron capture gamma cross section, one requires the dead time of the using detector system. In our case, the $4 \pi \mathrm{Ge}$ spectrometer has many detectors to be corrected for the dead times. In TOF experiments, the event rate has time structures. These made us difficult the dead time correction. In this paper, the dead time correction method was proposed.

\section{Outline of Our DAQ}

Figure 1 shows a conceptual design of our DAQ. The system consists of three Main ADC modules, three Fast Timing modules and a Coincidence module. Preamplifier outputs of the Ge detectors are directly sent to the Main ADC modules

${ }^{a}$ Presenting author, e-mail: kimura.atsushi@4@jaea.go.jp and the Fast Timing Modules. Outputs of BGO anti-Compton detectors are put only into the Fast Timing Modules.

On the Fast Timing module, input signals (both Ge detector and BGO) are shaped with a differentiation circuit, a pole-zero cancellation circuit, and amplification circuits. The shaped input signals become LVDS output signals with a Constant-Fraction Discriminator (CFD). The output LVDS signals of CFDs are input to the Coincidence Module.

On the Coincidence Module, the Field Programmable Gate Array (FPGA) compares the preset coincidence condition to trigger patterns. If the coincidence pattern meets the trigger pattern, the Coincidence Module sends a trigger signal to the Main ADC modules. Because the program of the FPGA can be uploaded via VME-bus, the coincident condition can be freely changed to suit trigger conditions. In TOF experiments, the Coincidence Module also measure and stores the delay time of the event from the start signal of neutron emission. We can measure the time with a resolution of $5 \mathrm{~ns}$ ranging up to $84 \mathrm{~ms}$.

The Main ADC Module has 16 input channels and each channel has analog circuits for differentiation, pole-zero cancellation, integrating, amplification and offset adjustment. The shaped output signals are digitized with 16 ADCs (12 bit $40 \mathrm{MHz}$ ), and the digitized data is stored in First In First Out memory (FIFO). When a trigger signal from the Coincidence Module comes, the FIFO sends the digitized data to a Digital Signal Processor (DSP). The pulse height is calculated by using the DSP and the calculated value is sent to a Dual Port Memory via another DSP for control. The data is read out from the Dual Port Memory with the VME-bus.

\section{Performance of this DAQ}

\subsection{Energy resolution at 1.33-MeV}

The energy resolution for $1.33-\mathrm{MeV}$ gamma rays was measured with a clover detector which is a part of the $4 \pi \mathrm{Ge}$ spectrometer, using a ${ }^{60} \mathrm{Co}$ gamma source.

For the peak height analysis of the Main ADC Module, two different kind of DSP program were used. One is the 


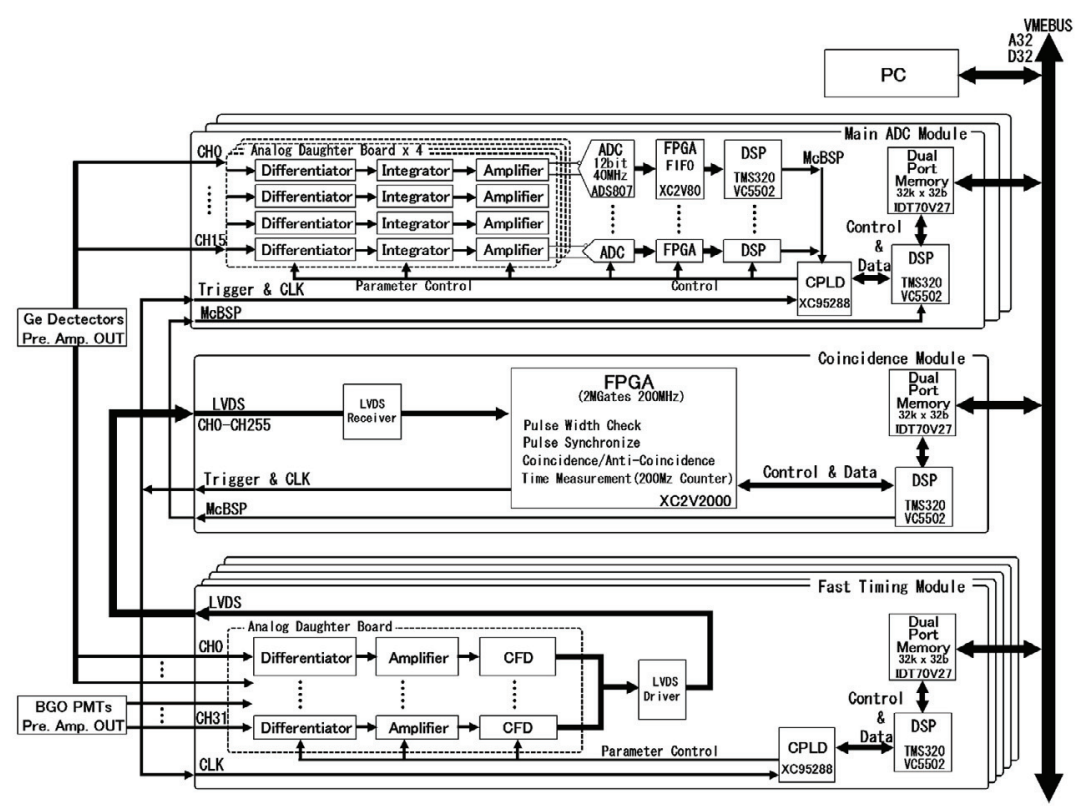

Fig. 1. Conceptual design of the Data Acquisition System for the $4 \pi$ Ge spectrometer [2].

Table 1. FWHMs of the full energy peak at $1.33 \mathrm{MeV}$ which were measured with a clover detector. These FWHMs were obtained with FIR Filtering or MWD Filtering. Energy resolution with a conventional multi channel analyzer (2100C MCA, Laboratory equipment, with a 572A Amplifier, ORTEC) was also measured in comparison.

\begin{tabular}{llll}
\hline & $\begin{array}{l}\text { FIR Filtering } \\
(\mathrm{keV})\end{array}$ & $\begin{array}{l}\text { MWD Filtering } \\
(\mathrm{keV})\end{array}$ & $\begin{array}{l}\text { MCA + Main } \\
\text { Amp. }(\mathrm{keV})\end{array}$ \\
\hline Ch1 & 2.57 & 2.52 & 2.40 \\
Ch2 & 2.48 & 2.42 & 2.30 \\
Ch3 & 2.62 & 2.54 & 2.43 \\
Ch4 & 2.41 & 2.39 & 2.31 \\
\hline
\end{tabular}

FIR Filtering method and the other was with Moving Window Deconvolution (MWD) filtering [3] (filter length: $2 \mu \mathrm{s}$ ) method. To evaluate energy resolution of this system, we also measured the gamma-ray spectrum with a conventional multi channel analyzer (2100C MCA, Labolatory equipment) with an amplifier (572A Amplifier, ORTEC). The results are shown in table 1 . The values are almost the same for MCA and the new system, and good energy resolution could be achieved with short shaping time.

\subsection{Energy resolution at high energy region}

To test the energy resolution of the DAQ system at the high energy region with the ${ }^{14} \mathrm{~N}(\mathrm{n}, \gamma){ }^{15} \mathrm{~N}$ reaction, we installed the $4 \pi$ Ge spectrometer on the cold neutron beam line C2-3-2 at a research reactor JRR-3M in Japan Atomic Energy Agency [4]. The ${ }^{14} \mathrm{~N}$ target was a 500-mg melamine resin enclosed with sheets of FEP film. Coincident prompt gamma-rays were measured for 3 hours. The energy range of the measured

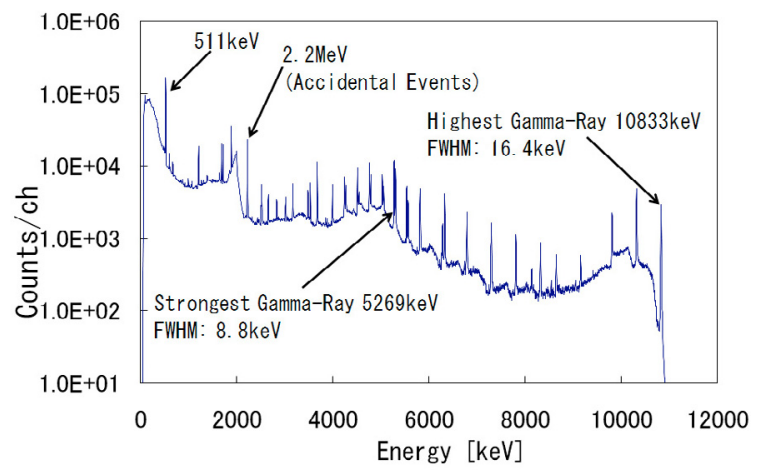

Fig. 2. A prompt gamma-ray energy spectrum of ${ }^{14} \mathrm{~N}$. The sample was 500-mg melamine resin enclosed with sheets of FEP film. The coincident prompt gamma-rays were measured for 3 hours.

spectra was $0-16 \mathrm{MeV}$ with $8 \mathrm{k}$ channels. The coincidence gate time was set to $800 \mathrm{~ns}$.

Figure 2 shows one measured prompt gamma-ray spectrum measured with crystal No. 1 of the clover detector No. 1. The two most intense gamma rays are at $5269-\mathrm{keV}$ and 5298-keV. Photo peaks, single escape peaks, and double escape peaks are clearly observed. The energy resolution is $8.8 \mathrm{keV}$ and $16.4 \mathrm{keV}$ in FWHM, for $5269-\mathrm{keV}$ and $10.83-$ $\mathrm{MeV}$ prompt gamma-rays, respectively. Those energy resolutions are good enough for our experiments. The $2223-\mathrm{keV}$ peak originating the $\mathrm{H}(\mathrm{n}, \gamma) \mathrm{D}$ background reaction was clearly observed, which is obviously accidental.

\subsection{Time resolution}

As mentioned above, time resolution is an important property of the DAQ system for collecting cascade gamma emission in a neutron capture reaction with good signal to noise ratio. 


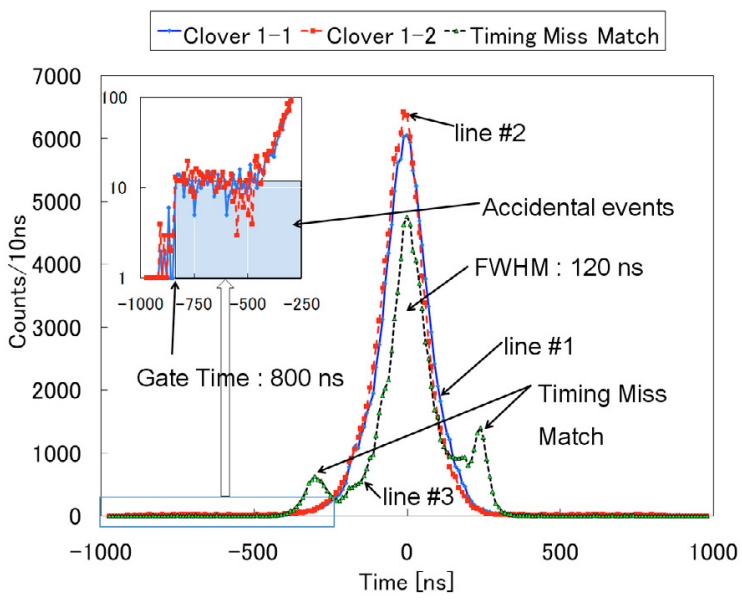

Fig. 3. Time dependence between a crystal and the other crystals. The line \#1 shows the time dependence of the channel No. 1 of the clover detector No. 1. The line \#2 shows that of the channel No. 2. And the line \#3 shows a case in which timing of a few crystals were miss-matched.

To test the time resolution of our DAQ, we measured the time delay of CFD signals to the trigger signal for each detector in the Coincidence Module. In this measurement, we measured coincidence prompt gamma-rays from ${ }^{14} \mathrm{~N}$ with the same setup given in the previous section.

Figure 3 is a result of the measurement. The horizontal axis is the time difference between the trigger signal and the detector signals. The vertical axis is event numbers for each time difference bin. Line \#1, and 2 denote different crystals. Both histograms indicate that the timing signals between crystals were well synchronized. The line \#3 shows a case in which signals of a few crystals in the Coincidence module have delay. Since FPGA on the Coincidence module has a logic delay, we can easily adjust the signal delay. With the histogram, we can easily estimate the ratio of the coincidence (true) events to the accidental (noise) events.

The FWHMs of these peaks were about $120 \mathrm{~ns}$. The FWHM with a multi channel analyzer (2100C MCA, Laboratory equipment) and a time to analog converter (TAC 567, ORTEC) was about $110 \mathrm{~ns}$. The both values are almost the same. It means that our DAQ system has a good time resolution as the conventional one.

\subsection{Development of the dead time correction method}

Because dead time is strongly dependent on many factors, such as detector configuration, beam strength, event rate, data transfer rate and so on, it is difficult to correct the dead time for each detector.

In this report we propose a new dead time correction method for such a many detector system as the $4 \pi$ Ge spectrometer with a random pulse generator. In this method, we input the random pulse to the every pre-amplifier of the Ge crystals via test inputs. Both the pulsar inputs and the other pulses originated from prompt gamma rays are measured with a DAQ. The number of the random pulses is counted with another system consisting of a fast timing amplifier, a CFD, and a counter. Because dead time affect similarly both the pulsar inputs and the measured promt-gamma rays, we can calculate dead time by comparing the number of the inputs random pulses obtained with the counter with the peak count of the pulser measured with our DAQ.

This method has been applied to TOF neutron capture cross section measurement experiments carried out with the $4 \pi \mathrm{Ge}$ spectrometer at the electron linear accelerator (LINAC) of Kyoto University Research Reactor Institute. The flight path length is about $10 \mathrm{~m}$. Random pulses were made with a noise pulsar (620 Noise Pulsar, Canberra) and a pulse generator (PB5 pulse generator, $\mathrm{BNC}$ ) and they were countered with a timer and counter (871 Timer and Counter, ORTEC).

\subsubsection{Test of the dead time correction method with ${ }^{152}$ Eu gamma sources}

To check the logic of this method, activity of a ${ }^{152} \mathrm{Eu}$ gamma source $(221 \mathrm{kBq})$ was calculated using another ${ }^{152} \mathrm{Eu}$ gamma source $(3.8 \mathrm{kBq})$ as a standard.

In a measurement with the standard source, the value of the photo peak at $1408-\mathrm{keV}$ from $\left(N_{\gamma s}\right)$ is calculated from the following equation

$$
N_{\gamma s}=S_{s} \times \varepsilon_{1408} \times I_{1408} \times L_{S} .
$$

Here, $S_{S}$ is the activity of the standard source, $\varepsilon_{1408}$ is the absolute photo peak efficiency of $1408-\mathrm{keV}$ gamma ray, $I_{1408}$ is the $1408-\mathrm{keV}$ gamma-ray emission intensity, and $L_{S}$ is the live time in the measurement with standard gamma source.

In this method, because the dead time affects similarly both the pulser inputs and the measured promt-gamma rays, the live time is expressed in following equation

$$
L_{S}=\frac{N_{S P D}}{N_{S P C}} .
$$

Here, $N_{S P C}$ is the number of the inputs pulses counted with ORTEC 871 , and $N_{S P D}$ is the peak count of the pulser measured with our DAQ in this measurement.

In a measurement with the other (stronger) ${ }^{152} \mathrm{Eu}$ gamma source, number of the photo peak at $1408-\mathrm{keV}\left(N_{\gamma T}\right)$ is expressed, in the same way.

$$
N_{\gamma T}=S_{T} \times \varepsilon_{1408} \times I_{1408} \times L_{T}=S_{T} \times \varepsilon_{1408} \times I_{1408} \times \frac{N_{T P D}}{N_{T P C}} .
$$

Here, $S_{T}$ is activity of the stronger source, $L_{T}$ is live time, $N_{T P C}$ is the number of the input pulses, and $N_{T P D}$ is the peak count of the pulser measured with our DAQ in the measurement with the stronger gamma source.

From equations (1)-(3), $\varepsilon_{1408}$ and $I_{1408}$ are cancelled and the activity of the stronger source is calculated easily from equation (4), with dead time correction.

$$
S_{T}=\frac{N_{\gamma T} \times L_{S}}{N_{\gamma S} \times L_{T}} \times S_{S}=\frac{N_{\gamma T} \times N_{S P D} \times N_{T P C}}{N_{\gamma S} \times N_{S P C} \times N_{T P D}} \times S_{S} .
$$

The measurement conditions and results are shown in table 2. Using this method, the activity of the stronger gamma source 
Table 2. The measurement condition and results. Peak counts of the pulser and $1.41 \mathrm{MeV}$ gamma-ray is shown at the center crystal of the cluster detector No. 1.

\begin{tabular}{lll}
\hline Gamma-ray source (kBq) & 3.77 & 221 \\
Measurement Time (sec) & 6771 & 927 \\
Event Rate (kevent/sec) & 1.2 & 20.7 \\
Data Transfer Rate (kByte/sec) & 110 & 570 \\
Pulse Count (Counter) & 2339636 & 381124 \\
Pulse Count (DAQ) & 2126336 & 134790 \\
Peak Count @ 1.33 MeV & 19024 & 5701 \\
Dead Time (\%) & 9.1 & 64.6 \\
Calculated source strength (kBq) & $-\ldots . .-\ldots$ & 224 \\
\hline
\end{tabular}

was calculated with high precision. This result shows that dead time can be corrected with this method. Although the event rate for the weaker gamma source was low enough, the dead time was more than $9 \%$. This is because the data transfer rate caused by the pulser is more than $100 \mathrm{kByte} / \mathrm{sec}$ (pulser rate was about $400 / \mathrm{sec}$ ) and this was a bottleneck of this measurement.

\subsubsection{Test of dead time correction method on TOF experiments}

To test this method in TOF experiments, we measured prompt gamma-rays from ${ }^{35} \mathrm{Cl}$. Target was salt powder $(\mathrm{NaCl} 42 \mathrm{~g}$ ) and measurement has been carried out for 3 hours with an electron energy of $30 \mathrm{MeV}$, a beam current of $30 \mu \mathrm{A}$, a pulse rate of $100 \mathrm{~Hz}$ and a pulse width of $0.1 \mu \mathrm{s}$.

Figure 4 shows a gamma-ray spectrum measured with the center crystal of the cluster detector No. 1. A peak originated from the pulse generater and the other many photo-peaks of prompt gamma rays are clearly observed. Figure 5 shows TOF spectrum of the prompt gamma rays and live time obtained with the same crystal. The live time was calculated from a TOF spectrum which was gated on the pulser peak in figure 4, and the TOF spectrum of the prompt gamma rays was ungated spectrum. It is clearly observed that the live time decreases as the event rate increases. This result shows that, on the TOF experiments, dead time can be corrected with this method.

\section{Summary}

We have described the performance of a new data acquisition system for the $4 \pi$ Ge spectrometer with digital signal processing techniques. In the tests with the spectrometer, we have obtained enough energy resolution and time resolution. We

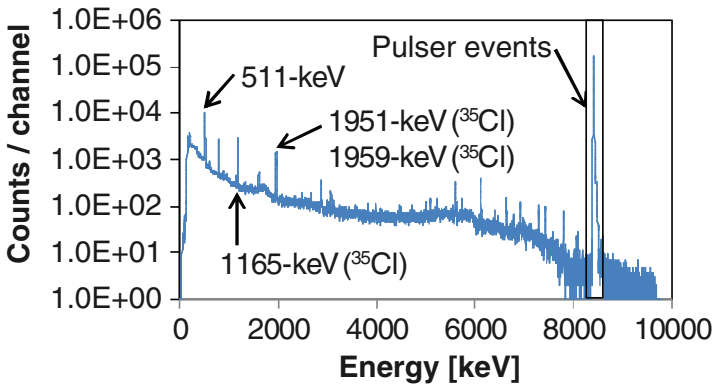

Fig. 4. A gamma-ray energy spectrum measured with the center crystal of the cluster detector No. 1. The sample was salt powder ( $\mathrm{NaCl}: 42 \mathrm{~g}$ ).

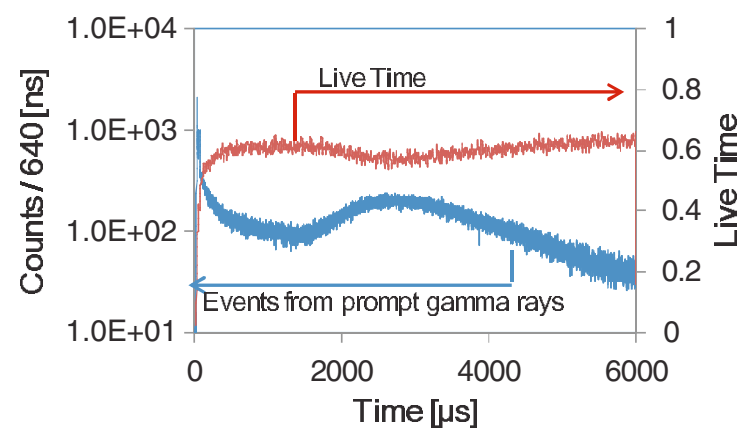

Fig. 5. TOF spectrum and live time at the center crystal of the cluster detector No. 1. Target was set on the $10 \mathrm{~m}$ neutron beam line.

have also developed a dead time correction method for the multiple gamma-ray detection method. These results show that our DAQ is a powerful system for the TOF experiments with a large germanium detector array.

This work was supported by the Ministry of Education, Culture, Sports, Science and Technology in Japan under the project "Fundamental R\&D on Neutron Cross Sections for Innovative Reactors using Advanced Radiation Measurement Technology".

\section{References}

1. J. Goto et al., in Proceedings of the International Conference on Nuclear Data for Science and Technology, 2004, edited by R.C. Haight et al. (AIP Conf. Proc. 769), p. 788.

2. A. Kimura et al., in Proceedings of the International Conference on Nuclear Data for Science and Technology, 2004, edited by R.C. Haight et al. (AIP Conf. Proc. 769), p. 792.

3. A. Georgiev et al., IEEE Trans. Nucl. Sci. 41, 1116 (1994).

4. http://rrsys.tokai-sc.jaea.go.jp/ (Japanese). 